\title{
Adesão da equipe de enfermagem ao protocolo de cirurgia segura
}

\author{
Adherence of the nursing team to safe surgery protocol \\ Adherencia del equipo de enfermería a lo protocolo de cirugías segura
}

Tatiana Menezes Noronha Panzetti ${ }^{1 *}$, Jéssica Maria Lins da Silva ${ }^{1}$, Lidiane Assunção de Vasconcelos ${ }^{1}$, Márcia Andréa da Gama Araújo1, Virgínia Mercês Lara Pessoa Oliveira1, Francinéa de Nazaré Ferreira de Castilho ${ }^{1}$, Giselle Castilho Maia ${ }^{1}$, Jéssica Da Silva Oliveira ${ }^{2}$, Tatiana Macedo Costa $^{2}$, Rosiane Pinheiro Rodrigues ${ }^{3}$, Aline Maria Pereira Cruz Ramos ${ }^{4}$

\section{RESUMO}

Objetivo: Analisar a adesão ao protocolo de cirurgia segura pela equipe de enfermagem como parte do processo de segurança assistencial. Métodos: Pesquisa qualitativa exploratória, realizada mediante entrevistas estruturadas com profissionais do centro cirúrgico de um Hospital Oncológico, com resultados analisados pelo método de Bardin. Realizada coleta de dados após liberação do Comitê de Ética em Pesquisa. Resultados: Foram entrevistados 26 participantes, 23 mulheres $(88,46 \%)$ e 3 homens $(11,54 \%)$, a média de idade foi 42 anos, com formação entre 5-25 anos e atuação 5-23 anos, sendo 1-21 em centro cirúrgico. Destes, 19 possuem somente um vínculo empregatício. Decorreram quatro categorias: Adesão da equipe de enfermagem ao protocolo de cirurgia segura; Aplicação do protocolo de cirurgia segura na instituição; Percepção da equipe de enfermagem sobre os pontos positivos e negativos na implementação do protocolo de cirurgia segura; Necessidades e contribuições a adesão ao protocolo de cirurgia segura. Conclusão: $O$ estudo demonstrou os fatores que influenciam na adesão da equipe ao protocolo sendo fundamental para embasamento de pesquisas futuras e elaboração de novas tecnologias na saúde.

Palavras-Chave: Equipe de enfermagem, Segurança do paciente, Centros cirúrgicos.

\section{ABSTRACT}

Objective: To analyze adherence to the safe surgery protocol by the nursing staff as part of the care security process. Methods: Qualitative exploratory research, conducted through structured interviews with professionals from the operating room of an Oncology Hospital, with results analyzed by the Bardin method. Data collection was performed after the Research Ethics Committee was released. Results: Twenty-six participants were interviewed, 23 women (88.46\%) and 3 men (11.54\%). The average age was 42 years old, with 5-25 years old and 5-23 years old. 21 in the operating room. Of these, 19 have only one employment relationship. Four categories resulted: Nursing staff adherence to the safe surgery protocol; Application of safe surgery protocol in the institution; Perception of the nursing staff about the positive and negative points in the implementation of the safe surgery protocol; Needs and contributions to adherence to safe surgery protocol. Conclusion: The study demonstrated the factors that influence the adherence of the team to the protocol, being fundamental for supporting future research and elaboration of new health technologies.

Keywords: Nursing staff, Patient safety, Operating room.

1 Universidade Estadual do Pará (UEPA), Belém - PA. *E-mail: tnpanzetti@hotmail.com

2 Faculdade Integrada Brasil Amazônia (FIBRA), Belém - PA.

3 Universidade Federal Fluminense (UFF), Niterói - RJ.

${ }^{4}$ Universidade Federal do Pará (UFPA), Belém - PA.

SUBMETIDO EM: 11/2019

ACEITO EM: 12/2019

PUBLICADO EM: 2/2020

REAS/EJCH | Vol.12(2) | e2519 | DOI: https://doi.org/10.25248/reas.e2519.2020 Página 1 de 8 


\section{RESUMEN}

Objetivo: analizar el cumplimiento del protocolo de cirugía segura por parte del personal de enfermería como parte del proceso de seguridad de la atención. Métodos: Investigación exploratoria cualitativa, realizada a través de entrevistas estructuradas con profesionales del quirófano de un Hospital de Oncología, con resultados analizados por el método Bardin. La recopilación de datos se realizó después de la publicación del Comité de Ética en Investigación. Resultados: Entrevistamos a 26 participantes, 23 mujeres (88.46\%) y 3 hombres (11.54\%), la edad promedio fue de 42 años, con entrenamiento entre 5 y 25 años y actuando de 5 a 23 años, con $1-21$ en el quirófano. De estos, 19 tienen solo una relación laboral. Se obtuvieron cuatro categorías: adhesión del personal de enfermería al protocolo de cirugía segura; Aplicación del protocolo de cirugía segura en la institución; Percepción del personal de enfermería sobre los puntos positivos y negativos en la implementación del protocolo de cirugía segura; Necesidades y contribuciones a la adherencia al protocolo de cirugía segura. Conclusión: El estudio demostró los factores que influyen en la adhesión del equipo al protocolo, siendo fundamentales para apoyar la investigación futura y la elaboración de nuevas tecnologías de salud.

Palabras clave: Personal de enfermería, Seguridad del paciente, Sala de operaciones.

\section{INTRODUÇÃO}

O termo adesão tem sido usado para denominar o grau de coincidência entre os comportamentos do indivíduo e atribuir sua funcionalidade. No contexto da saúde, ele pressupõe uma parceria entre quem cuida e quem é cuidado, tendo início quando o paciente e o profissional seguem as orientações padrões. Deste modo, o desenvolvimento de ferramentas que forneçam informação, tais como checklists, promovem a identificação precoce de problemas mais frequentes para o planejamento da assistência de enfermagem, durante a internação hospitalar, desenvolvimento do plano de alta e orientação sobre cuidados em domicílio. (ALPENDRE FT, et al., 2017).

Entretanto, observa-se uma preocupação com a falta de concordância entre as orientações, os treinamentos e recomendações. Esta problemática tende a se intensificar caso outros aspectos, comuns no ambiente hospitalar, se intensifiquem, tais como: condições da ambiência, deficiência de materiais e equipamentos, falhas na comunicação, desgaste físico e emocional dos trabalhadores e limitações da equipe médica quanto a compreensão do trabalho da enfermagem (MARTINS FZ, et al., 2016).

Portanto, a adesão ao checklist do protocolo assistencial de cirurgia segura, visa diminuir o atrito provocado por situações inesperadas e aumentar a segurança da equipe, da instituição e, principalmente, do paciente (PAIXAO DPSS, et al., 2018). Além disso, esta tecnologia, se usada pela equipe de forma adequada, pode constituir-se um espaço para ensino, aprendizagem e produções científicas (SOARES MI, et al., 2015).

De acordo com a Organização Mundial de Saúde, as ações de segurança ao paciente são fundamentais para a prática da assistência, devendo ser disseminadas objetivando o desenvolvimento de conhecimentos e estratégias para vislumbrar a completa segurança ao paciente cirúrgico. Dessa forma, destaca que as principais medidas a serem tomadas pelos profissionais da área da saúde neste quesito incluem a prevenção de eventos adversos; a detecção destes, caso ocorram; e a minimização de complicações com intervenções resolutivas e eficazes (AVELAR MCQ, et al., 2011). A partir disso, desenvolveu-se o conceito atual de segurança do paciente, como sendo a redução de riscos e danos desnecessários ao mínimo aceitável recebendo, para tal feito, auxílio dos avanços na área da saúde e das mudanças assistenciais (SILVA AT, et al., 2016). Outrossim, grande parte das lacunas encontradas na segurança do paciente, em especial no paciente cirúrgico, decorre de iatrogenias cometidas pela própria equipe hospitalar, o que pode acarretar complicações graves e permanentes aos que estão sob seus cuidados. Dessa forma, destaca-se o papel do enfermeiro como responsável pelo controle da assistência prestada ao paciente em todas as fases do processo saúde-doença, bem como da importância da implementação de um cuidado padronizado (SILVA AT, et al., 2016). 
A Sistematização da Assistência de Enfermagem Perioperatória (SAEP) desenvolveu-se com o objetivo de especificar e qualificar os cuidados de enfermagem, voltando-os a pacientes cirúrgicos. Deste modo, sua implementação visa promover a assistência integral, continuada, participativa, individualizada, documentada e avaliada, no qual o paciente é singular e a assistência de enfermagem é uma intervenção conjunta que promove a continuidade do cuidado, além de proporcionar a participação da família do paciente e possibilitar a avaliação da assistência prestada (FONSECA RMP, et al., 2009).

Assim, o enfermeiro do centro cirúrgico tem papel fundamental em suas atividades diárias, contribuindo no gerenciamento do cuidado de enfermagem no perioperatório (BIANCHI ERF e CARVALHO R, 2016). As atividades gerenciais são ações com a finalidade de assegurar a qualidade da assistência de enfermagem e o bom funcionamento da instituição, considerando que o foco no centro cirúrgico é centrado no paciente, o profissional deve estar preparado, além de treinar e habilitar sua equipe para o trabalho conjunto, visando uma assistência humanizada com vistas à segurança e ao bem-estar do paciente cirúrgico (POSSARI JF, 2014). Além disso, deve adquirir as competências necessárias para prever as necessidades que possivelmente estarão afetas após o procedimento cirúrgico, devendo atentar-se as etapas desenvolvidas pelos profissionais do centro ao longo da assistência, visando um auxílio integral e resolutivo (SANTOS NCM, 2010).

A inquietação pelo estudo surgiu durante práticas educativas acadêmicas em um hospital de grande porte no município de Belém/Pará, ao qual observou-se que o protocolo de cirurgia segura é implementado, porém os profissionais de saúde não aderem integralmente ao preenchimento do checklist. Em vista disso, objetivouse através deste estudo, analisar a adesão ao protocolo de cirurgia segura pela equipe de enfermagem.

\section{MÉTODOS}

Pesquisa qualitativa do tipo exploratória descritiva, realizada nas dependências cirúrgicas de um hospital público oncológico no estado do Pará, com o consentimento da referida instituição para a publicação dos resultados. Para a realização do estudo, delimitaram-se as seguintes questões norteadoras: como ocorre a adesão ao protocolo de cirurgia segura pela equipe de enfermagem como parte integrante do protocolo? Qual a percepção dos profissionais de enfermagem em relação à aplicação do protocolo de cirurgia segura?

A partir disso, foram entrevistados 26 participantes da equipe de enfermagem, sendo três enfermeiros e vinte e três técnicos de enfermagem. Os dados foram coletados no mês de agosto de 2018. Selecionaram-se os seguintes critérios de inclusão para escolha da amostra: enfermeiros e técnicos de enfermagem com pelo menos 6 meses de experiência profissional anterior em centro cirúrgico, maiores de 21 anos de ambos os sexos, atuantes no mínimo por 3 meses no centro cirúrgico da instituição estudada. Quanto à exclusão: excluídos outros profissionais da área da saúde.

As entrevistas individualizadas foram aprazadas conforme disponibilidade dos participantes em relação ao dia e ao horário. Durante a entrevista, foram expostos os objetivos do estudo, a necessidade de gravação da entrevista em áudio digital, leitura do Termo de Consentimento Livre e Esclarecido (TCLE) e Termo de Gravação de Voz, ressaltando a garantia do anonimato e privacidade aos participantes. Para a coleta de dados utilizou-se um roteiro semiestruturado com questões sobre o perfil socioprofissional e outras específicas, relativas à adesão da equipe de enfermagem ao protocolo de cirurgia segura, ocorrendo até a saturação do estudo, que se deu com a repetição das respostas e seus sentidos. A análise foi realizada de acordo com a técnica de análise de conteúdo de Bardin. Este tipo de metodologia configura-se através de um conjunto de técnicas de análises comunicativas, que juntas visam a compreensão, a descrição e a compilação dos conteúdos estudados (CAMPOS CJG, 2004).

Deste modo, o estudo delineou-se a partir das fases de análise de conteúdo, que envolveu a pré-análise das entrevistas transcritas na íntegra, exploração do material, com tratamento dos resultados, dedução e interpretação, permitindo o surgimento de categorias e subcategorias a partir da identificação das expressõeschaves e ideias principais. A pesquisa obedeceu a Resolução №466/12 do Conselho Nacional de Saúde do Ministério da Saúde, que dispõe sobre Diretrizes e Normas Regulamentadoras para pesquisas envolvendo 
seres humanos. O projeto foi apreciado e aprovado pelo Comitê de Ética em Pesquisa (CEP) da Faculdade Integrada Brasil Amazônia (FIBRA), sob o protocolo no CAAE 91194118.7.0000.8187.

\section{RESULTADOS E DISCUSSÃO}

Foram entrevistados vinte e seis (26) participantes, prevalecendo o sexo feminino correspondente a vinte e três mulheres (23) $(88,46 \%)$ e a três (3) homens $(11,54 \%)$, a idade variou de trinta (30) a cinquenta e quatro (54) anos, sendo a média quarenta e dois (42) anos. Quanto ao tempo de formação variou de cinco (5) a vinte e cinco (25) anos, com média de quinze (15) anos. Em paralelo ao tempo de formação, o tempo de atuação na enfermagem variou de cinco (5) a vinte e três (23) anos. Os dados levantados sobre outros vínculos empregatícios mostraram que 19 participantes apresentam somente um vínculo empregatício, sendo esta na instituição estudada, e sete (7) possuem outros vínculos laborais. Quanto ao tempo de trabalho em centro cirúrgico, variou de um (1) a vinte e um (21) anos, mostrando o contato e experiência com a aplicação ao protocolo de cirurgia segura no local de trabalho da equipe de enfermagem estudada. A seguir, serão apresentadas as categorias temáticas que emergiram da análise das falas dos participantes.

\section{1) Adesão da equipe de enfermagem ao protocolo de cirurgia segura}

Nessa categoria descreveu-se a adesão de três participantes que possuem relação ao tema "adesão ao protocolo". Entre os profissionais entrevistados apenas dois técnicos de enfermagem não tinham conhecimento algum sobre o protocolo. Ademais, a adesão foi relatada com a expressão "Boa", apesar do aumento de "papéis" a preencher na rotina do serviço.

"Houve uma resistência por parte de alguns colegas, com a alegação de que seria mais um papel para ser preenchido. Hoje temos uma boa aceitação." (Tarde - ETécnico de enfermagem).

"A adesão tem sido boa, aceitável e realizada conforme a rotina do hospital." (Manhã - $D$ - Técnico de enfermagem).

"Foi Boa, apesar de aumentar os papéis." (Manhã - B - Técnico de enfermagem). "Não sei" (Manhã - N- Técnico de enfermagem).

"Não tenho como responder" (Tarde - I - Técnico de enfermagem).

Dois participantes demonstraram resistência quanto a implementação do protocolo por meio de expressões associadas ao processo de adesão do checklist.

"No início houve bastante resistência da equipe por ser mais um papel a ser preenchido, após as reuniões e os treinamentos, houve uma melhor adesão pela equipe." (Tarde $-H$ - Técnico de enfermagem).

"No início tinha bastante resistência, mas hoje já aderiram ao protocolo." (Tarde - K - Enfermeiro).

E ainda, houve a descrição que a adesão pelos enfermeiros é integral, tendo em vista que o protocolo frequentemente é preenchido pelo profissional, ressaltando que ainda precisa melhorar a adesão pela equipe técnica de enfermagem, o que contradiz os resultados das entrevistas dos técnicos.

"Adesão pelos enfermeiros é total e pela equipe técnica de enfermagem ainda precisa melhorar." (Manhã - G - Enfermeiro).

Assim, observou-se que a maioria dos técnicos de enfermagem descreveu possuir adesão ao protocolo de cirurgia segura e uma minoria apresentou resistência, alegando que seria mais um papel a ser preenchido, contudo, com a adesão majoritária, pode-se concluir que os planejamentos cirúrgicos apresentam bom embasamento. 


\section{2) A aplicação do protocolo de cirurgia segura na instituição}

Nesta categoria descreveu-se a aplicação do protocolo de cirurgia segura no momento da admissão do paciente no centro cirúrgico, dividido nas etapas: check-in, time out e check-out. Os Enfermeiros e Técnicos expressaram que a aplicação se dá através do preenchimento dos impressos normatizados na instituição, como identifica-se a seguir:

"Através de impressos que são preenchidos pelo enfermeiro com a ajuda dos técnicos, com perguntas ao paciente e toda equipe da sala cirúrgica". (Manhã - E Técnico de enfermagem).

"Ocorre através de um impresso para cada paciente em todas as cirurgias e no quadro de aviso de cirurgia segura das salas operatórias." (Manhã - F - Técnico de enfermagem).

"Realizado pelo enfermeiro do plantão, com a equipe técnica de enfermagem". (Manhã - G - Enfermeiro).

"Ao receber o paciente, o enfermeiro inicia o preenchimento do protocolo e o técnico finaliza após o término da cirurgia". (Tarde - $H$ - Técnico de enfermagem).

Outros ainda agregaram que houve bastante resistência pela equipe médica de cirurgiões e anestesistas na aplicação e operacionalização do protocolo.

"Bastante resistência principalmente da equipe médica". (Tarde - E - Técnico de enfermagem).

"Ocorre entre a equipe de enfermagem, faltando um pouco de colaboração dos outros profissionais". (Manhã - O - Técnico de enfermagem).

Portanto, observou-se nas falas que a aplicação ocorre através do preenchimento de impressos que fazem parte do prontuário do paciente, mas acredita-se que a resistência e dificuldades no preenchimento seja devido à falta de capacitação e sensibilização quanto a política de segurança do paciente cirúrgico para toda a equipe cirúrgica e da grande rotatividade da equipe de profissionais. Entretanto, observou-se o empenho em seguir o protocolo e a preocupação com o preenchimento correto como forma de promover a segurança do paciente.

\section{3) Percepção da equipe de enfermagem sobre os pontos positivos e negativos na implementação do protocolo de cirurgia segura:}

Observou-se que os pontos positivos destacados pelos entrevistados sobre o protocolo foram bastante expressivos, como: comunicação entre os profissionais, organização de prontuários e redução de possíveis erros, os quais percebe-se nas falas a seguir:

"O protocolo de cirurgia segura, veio para beneficiar o paciente e evitar erros, pois isso é muito comum, junto com as infecções e problemas nos materiais cirúrgicos." (Manhã - A - Técnicos de enfermagem)

"Positivos: a identificação correta do paciente, comunicação entre os profissionais melhorou, assegurar a cirurgia local, lateralidade, prescrição do paciente [...]" (Manhã - C - Técnicos de enfermagem)

"Maior segurança ao paciente, organização dos prontuários, mas direcionamento da assistência [...]" (Manhã - E - Técnicos de enfermagem)

"Segurança do paciente e da equipe, redução de possíveis erros, e controle nos procedimentos realizados [...]" (Tarde - E - Técnicos de enfermagem)

"Foi criado mais um instrumento para a segurança do paciente, evitando erros durante e após o ato cirúrgico [...]" (Tarde - H - Técnicos de enfermagem) 
Os pontos negativos descritos apontam dificuldade na adesão ao preenchimento do protocolo e na comunicação com a equipe médica e anestesistas:

"[...] mais participação da equipe cirúrgica na hora do checklist." (Manhã - C Técnico de enfermagem)

"[...] dificuldade na adesão de alguns profissionais, número reduzido de enfermagem, para aplicação mais eficaz." (Tarde - E - Técnico de enfermagem)

"[...] Falta de adesão da equipe médica para com a cirurgia segura." (Tarde - H Técnico de enfermagem)

Portanto, existem desafios relacionados à implementação e execução do protocolo de cirurgia segura, sendo o principal a resistência da equipe médica em participar das etapas do protocolo. Além disso, destacase a necessidade do aumento de enfermeiros, devido à sobrecarga de atendimento no centro cirúrgico, para que o enfermeiro possa assistir com qualidade e integralidade o cliente cirúrgico.

\section{4) Necessidades e contribuições à adesão ao protocolo de cirurgia segura}

Diante dos desafios e dificuldades apresentadas pelos profissionais, houve aqueles que destacaram a necessidade de mudanças de paradigmas entre os profissionais, a partir do desenvolvimento de educação permanente, conforme relatos:

"O que precisa é de uma união maior da enfermagem, assim o trabalho prestado, a segurança, a assistência ao paciente só tende a melhorar cada vez mais." (Manhã $H$ - Técnico de enfermagem)

"A educação em serviço deve ser continua para toda a equipe." (Manhã - J Enfermeira)

"Falta uma melhor abordagem com a classe médica, para que ocorra sintonia na equipe multidisciplinar, assim dará segurança ao paciente." (Tarde - I - Técnico de enfermagem)

"Acredito que para ser implantando de forma completa o protocolo de cirurgia segura, necessitamos de uma quantidade maior de enfermeiros." (Tarde - K Enfermeira)

Portanto, na visão dos profissionais há necessidade de mudança na cultura interna no sentido de promover a segurança, comunicação e qualidade da assistência prestada pela equipe multiprofissional, com investimento em educação permanente, ampliação do conhecimento acerca da importância do protocolo, promoção do trabalho em equipe e divisão das responsabilidades.

A partir do exposto evidenciou-se que a equipe de enfermagem na maioria é do gênero feminino (23 participantes), a idade variou entre 30 a 54 anos, corroborando com o perfil da enfermagem brasileira na região Norte. Desta forma, torna-se obrigatório conhecer a estrutura e a dinâmica da enfermagem no Brasil, considerando que a categoria de enfermagem possui suas características próprias, trazendo consigo a maior categoria profissional do campo da saúde no Brasil, com papéis que continuamente vem sendo ampliados (BACKES DS, et al., 2012).

Diante dos diversos fatores relacionados ao trabalho dos profissionais de saúde, principalmente em relação ao cotidiano da equipe cirúrgica, observou-se que as maiores barreiras para aplicação do protocolo de cirurgia segura foram: pouca comunicação da equipe cirúrgica, não adesão ao preenchimento do protocolo pelos profissionais, especificamente cirurgiões e anestesistas.

Além de sobrecarga da equipe de enfermagem, a qual representa a categoria dos profissionais de saúde que se responsabilizam pelo preenchimento e aplicação de prontuários e protocolos assistenciais, justificando as falas encontradas. (FRANCO MTG, et al., 2012). Para tanto, visando a utilização correta do instrumento de checklist de cirurgia segura, a equipe deve ser capacitada e orientada, incentivando percepções individuais 
entre os membros da equipe cirúrgica sobre a importância de cada etapa do protocolo e influenciando diretamente sua implementação (FRAGATA JIG, 2010). Posto que esta padronização se trata de uma ferramenta moderna, que apoia e embasa a tomada de decisão dos profissionais, em especial do enfermeiro na qualidade de gestor do cuidado.

Além disso, possibilita corrigir as não-conformidades, permite que todos os trabalhadores prestem um cuidado padronizado para o paciente de acordo com princípios técnico-científicos, contribui para dirimir as distorções adquiridas nas práticas, proporciona maior satisfação da equipe e do usuário do serviço, ocasionando consequentemente maior segurança na realização de procedimentos, bem como o controle assistencial por toda a equipe e pela instituição (SALES CB, et al., 2018).

A comunicação na sala cirúrgica entre a equipe foi relatada como item a ser melhorado. Essas dificuldades comunicativas emergiram no conteúdo das entrevistas, expressas em falas como "[...] falta de adesão da equipe médica [...]", "resistência". O manual de cirurgia segura lançado pelo Ministério da Saúde determina que a ideia do protocolo é promover o trabalho em equipe, com abordagem multiprofissional, favorecendo a comunicação e a checagem de todos os elementos relacionados à assistência no período perioperatório. Portanto, nota-se a necessidade de investir na educação permanente com a equipe do centro cirúrgico, em especial equipe médica, visando maior interação entre os profissionais e maior adesão a essa ferramenta, corroborando, assim, o destacado na literatura acerca da importância da educação permanente para transformar e qualificar a atenção à saúde (CAMPOS KFC, et al., 2017). Bem como no incentivo a uma comunicação clara e objetiva entre os profissionais, visto a importância da comunicação como instrumento laboral dos trabalhadores de saúde e da necessidade de implementação da comunicação dialógica, pautada na relação de troca e intercâmbio de saberes que deve ocorrer no cenário do Sistema Único de Saúde, objetivando a melhora na qualidade assistencial aos usuários e seus familiares (CORIOLANO-MARINUS MWL, et al., 2014).

Ademais, houve profissionais que consideraram o protocolo de cirurgia segura como mais um papel a ser preenchido, como descrito em outras pesquisas nas quais $7 \%$ dos participantes não reconheceram a importância do protocolo de cirurgia segura. Outros estudos ratificaram este feito, destacando que nem sempre os profissionais percebem a importância de novos instrumentos e tecnologias empregadas para promoção da segurança do paciente, motivo pelo qual existe a necessidade de as instituições promoverem uma mudança cultural em torno do erro e suas representações, saindo de abordagens punitivas para aquelas de promoção do conhecimento (MENDES R, et al., 2016).

Assim, o protocolo não é apenas um instrumento para garantir a segurança ao paciente e sim um importante método de respaldo da equipe cirúrgica e da instituição (RODRIGUES RD, et al., 2012). Ao qual dará oportunidade de manter a organização assistencial no centro cirúrgico, identificação do paciente, lateralidade, segurança na quantidade e qualidade dos materiais, reserva sanguínea, profilaxia antimicrobiana, entre outros (ROTHROCK JC, 2007). Entretanto a comunicação na sala cirúrgica é algo que precisa ser melhorado, uma vez que muitos profissionais, como demostrado neste estudo, têm dificuldades em partilhar informações, interferindo diretamente no desempenho da equipe e no preenchimento correto e total do protocolo.

Portanto, estudos evidenciaram uma redução significativa nas taxas de mortalidade e complicações consequente dos erros cirúrgicos após a aplicação do protocolo de cirurgia segura, com melhorias na segurança do paciente cirúrgico em todo o período perioperatório, principalmente devido a mudança na comunicação da equipe cirúrgica. Promovendo, deste modo, uma assistência comprometida em assegurar a promoção, proteção de cuidados com a vida, bem-estar e saúde (GIRON MN, et al., 2013). Outrossim, salienta-se a necessidade do uso adequado do protocolo por profissionais, promovendo a compreensão da importância deste para o paciente. Além disso, percebe-se a importância da educação permanente para a eficiência e a eficácia de um cuidado amplo e seguro no centro cirúrgico, construindo uma nova consciência da realidade do pensar com a troca, a reciprocidade e a integração entre diferentes áreas, com aquisição de conhecimentos de forma contínua, possibilitando a resolução de problemas de forma global e abrangente (SILVA GM, et al., 2009). 


\section{CONCLUSÃO}

O estudo coincidiu com os objetivos iniciais, uma vez que proporcionou a análise acerca da adesão da equipe de enfermagem ao protocolo de cirurgia segura, promovendo a reflexão e sensibilização da equipe de enfermagem sobre a importância desses cuidados ao paciente no âmbito do centro cirúrgico. Possibilidades futuras envolvem a investigação sobre a adesão da equipe cirúrgica aos protocolos assistenciais, abrangendo um número maior de profissionais de saúde e de variadas categorias, para identificar possíveis problemas e soluções, com a finalidade de melhorar a segurança no ato cirúrgico, proporcionando a valorização do instrumento no processo de trabalho e auxiliando na elaboração de novas estratégias de aplicação, acompanhamento e avaliação da assistência cirúrgica hospitalar.

\section{REFERÊNCIAS}

1. ALPENDRE FT, et al. Cirurgia segura: validação de checklist pré e pós-operatório. Revista Latino Americana de Enfermagem, 2017; 25: e2907.

2. AVELAR MCQ, et al. Segurança do cliente e as ações frente ao procedimento cirúrgico. Revista Eletrônica de Enfermagem, 2011;13(2):347-54.

3. BACKES DS, et al. O papel profissional do enfermeiro no Sistema Único de Saúde: da saúde comunitária à estratégia de saúde da família. Ciência e saúde coletiva, 2012; 17 (1): 223-230.

4. BIANCHI ERF, CARVALHO R. Enfermagem no centro cirúrgico e recuperação. Barueri: Manole, 2ํㅡ. Ed. 2016 ; 428p.

5. CAMPOS CJG, et al. Método de análise de conteúdo: ferramenta para a análise de dados qualitativos no campo da saúde. Revista Brasileira de Enfermagem, 2004; 57 (5): 611-614.

6. CAMPOS KFC, et al. Educação permanente nos serviços de saúde. Escola Anna Nery, 2017; 21 (4): e20160317.

7. CORIOLANO-MARINUS MWL, et al. Comunicação nas práticas em saúde: revisão integrativa da literatura. Saúde e Sociedade, 2014; 23 (4): 1356-1369.

8. FONSECA RMP, PENICHE ACG. Enfermagem em centro cirúrgico: trinta anos após criação do Sistema de Assistência de Enfermagem Perioperatória. Acta Paulista de Enfermagem, 2009; 22 (4): 428-433.

9. FRAGATA JIG. Erros e acidentes no bloco operatório: revisão do estado da arte. Revista Portuguesa de Saúde Pública, 2010; 10:17-26.

10. FRANCO MTG, et al. Avaliação dos registros de enfermeiros em prontuários de pacientes internados em unidade de clínica médica. Acta Paulista de Enfermagem, 2012; 25 (2): 163-170.

11. GIRON MN, et al. O acolhimento no centro cirúrgico na perspectiva do usuário e a Política Nacional de Humanização. Revista Enfermagem UERJ, 2013; 21(esp.2):766-71.

12. MARTINS FZ, et al. Centro cirúrgico: desafios e estratégias do enfermeiro nas atividades gerenciais. Revista Gaúcha de Enfermagem, 2016; 37 (4): e56945.

13. MENDES R, et al. Promoção da saúde e participação: abordagens e indagações. Saúde em Debate, $2016 ; 40$ (108): 190-203.

14. PAIXAO DPSS, et al. Adesão aos protocolos de segurança do paciente em unidades de pronto atendimento. Revista Brasileira de Enfermagem, 2018; 71 (1): 577-584.

15. POSSARI JF. Centro Cirúrgico: Planejamento, Organização e Gestão. São Paulo: látria, 5ª Ed. 2014; $288 p$.

16. RODRIGUES RD, et al. Segurança do paciente: Check-list como ferramenta para a cirurgia segura. Centro $\begin{array}{lll}\text { Universitário UNanciscano, } & \text { UNIFRA, } & \text { Disponível }\end{array}$ em:http://www.unifra.br/eventos/jornadadeenfermagem/Trabalhos/3710.pdf. Acesso em: 25 de setembro de 2018.

17. ROTHROCK JC. Alexander: Cuidados de enfermagem ao paciente cirúrgico. Rio de Janeiro: Elsevier, 13ª Ed. 2007; $1272 p$.

18. SALES CB, et al. Protocolos Operacionais Padrão na prática profissional da enfermagem: utilização, fragilidades e potencialidades. Revista Brasileira de Enfermagem, 2018; 71 (1): 126-134.

19. SANTOS NCM. Centro cirúrgico e os cuidados de enfermagem. São Paulo: látria 6aㅡ Ed. 2010; 224p.

20. SILVA AT, et al. Assistência de enfermagem e o enfoque da segurança do paciente no cenário brasileiro. Saúde em debate, 2016; 40 (111): 292-301.

21. SILVA GM, et al. Educação continuada em enfermagem: uma proposta metodológica. Revista Brasileira de Enfermagem, 2009; 62 (3): 362-366.

22. SOARES MI, et al. Sistematização da assistência de enfermagem: facilidades e desafios do enfermeiro na gerência da assistência. Escola Anna Nery, 2015; 19 (1): 47-53. 